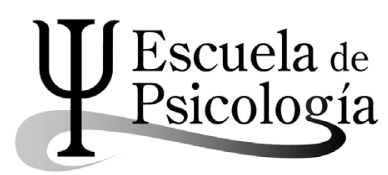

Wímb lu, Rev. electrónica de estudiantes Esc. de psicología, Univ. de Costa Rica. 10 (2): 19-37, 2015 / ISSN: 1659-2107

\title{
PERFIL POR COMPETENCIAS LABORALES $Y$ MODELO DE SELECCIÓN DE PERSONAL PARA EL CARGO TÉCNICO A EN GESTIÓN DE RECURSOS HUMANOS
}

\author{
Profile for job skills and recruitment model for the A Technical Management \\ Position in Human Resources
}

\author{
Maydel Fernández Alonso \\ Maydeé Vázquez Padilla \\ Gabriela María Dujarríc Bermúdez \\ Nadir Díaz Simón \\ Hansel Soto Hernández *
}

\begin{abstract}
Resumen: La presente investigación está centrada en la elaboración de un perfil de competencias laborales del cargo Técnico "A" en Gestión de Recursos Humanos de la Dirección General de Recursos Humanos (DGRH) de la Oficina del Historiador de la Ciudad de La Habana $(\mathrm{OHCH})$, y de un modelo de selección de personal para el cargo. La muestra está conformada por dos grupos de sujetos: informantes clave y expertos en el puesto de trabajo. Se siguió un enfoque mixto con técnicas cualitativas y cuantitativas, aplicadas en tres etapas. En el estudio se concluye que las competencias clave del cargo según la ponderación de los expertos, son: capacidad de organización, comunicación eficientepersuasiva, comportamiento ético, autocontrol emocional y gestión de información. Las técnicas que constituyen el modelo de selección están conformadas principalmente por pruebas de papel y lápiz, proyectivas, juego de roles y entrevistas.
\end{abstract}

Palabras clave: Gestión de Recursos Humanos, selección de personal, competencias laborales, perfil por competencias.

* Universidad de La Habana. Estudiantes de psicología. Cuba.

Correos electrónicos: maydel.fernadez@psico.uh.cu, maydee.vazquez@psico.uh.cu, gabriela. dujarric@psico.uh.cu, nadir.diaz@cneuro.edu.cu, hansel.soto@psico.uh.cu

** Colaboradores: MsC. Arianne Medina, Universidad de La Habana, Ing. Hansel Ruiz-Calderón, Dirección General de Recursos Humanos de la Oficina del Historiador de la Ciudad.

Recepción: 25/10/2014 Aceptación: 8/4/2015 


\begin{abstract}
This research is focused on developing a profile of job skills Technical office "A" in Human Resource Management Directorate General of Human Resources (DGRH) of the Office of the City Historian of Havana $(\mathrm{OHCH})$ and a model of recruitment for the post. The sample consists of two groups of subjects: key informants and experts in the workplace. A mixed approach with qualitative and quantitative techniques applied in three steps were followed. The study concludes that key competencies of charge according to the weighting of the experts, are: organizational skills, persuasive communication-efficient, ethical behavior, emotional self-control and information management. The techniques are model selection are mainly made up of paper and pencil tests, projective, role plays and interviews.
\end{abstract}

Key Words: Human Resources Management, recruitment, job skills, competency profile.

\title{
Introducción
}

La gestión por competencias tuvo su surgimiento a principios de la década de 1970, siendo su principal exponente David C. McClelland. En una de las investigaciones primigenias sobre el tema, realizada por este autor junto a Richard E. Boyatzis en la compañía estadounidense de telecomunicaciones American Telephone and Telegraph (AT\&T) vio la luz una de las definiciones más utilizadas para conceptualizar las competencias, la cual fue definida como: "Unas características subyacentes a la persona, que están causalmente relacionadas con una actuación exitosa en un puesto de trabajo" (1982; citado por Cuesta, 2000).

Desde el comienzo de los estudios sobre las competencias laborales existió una falta de consenso sobre su definición, situación que se mantiene hasta la actualidad. Así por ejemplo, para Boyatziz, (1982) y Spencer y Spencer (1993), es una característica subyacente en el individuo que está causalmente relacionada a un estándar de efectividad y/o un desempeño superior en un trabajo o situación. Para Mertens (2000) es la "aptitud de un individuo para desempeñar una misma función productiva en diferentes contextos y con base en los requerimientos de calidad esperados por el sector productivo". Este autor plantea que dicha aptitud se alcanza a partir de la apropiación y desarrollo de conocimientos, habilidades y capacidades, los cuales se expresan en el saber, el hacer y el saber hacer.

Según Cuesta (2000) la competencia es un punto de conexión entre los comportamientos de las personas y los objetivos estratégicos de la organización a través del trabajo concreto y es aquí donde ellas se conectan con la estrategia de Gestión de Recursos Humanos (GRH), contribuyendo a la innovación de este proceso organizacional.

Consecuentemente con la Legislación Laboral Cubana (Resolución No. 21/99 del Ministerio del Trabajo y Seguridad Social de la República de Cuba) la Norma Cubana 3000:2007 ${ }^{1}$ define las competencias como:

Conjunto sinérgico de conocimientos, habilidades, experiencias, sentimientos, actitudes, motivaciones, características personales, y valores, basado en la idoneidad 
demostrada, asociado a un desempeño superior del trabajador y de la organización, en correspondencia con las exigencias técnicas, productivas y de servicios. Es requerimiento esencial que esas competencias sean observables, medibles y que contribuyan al logro de los objetivos de la organización.

La gestión por competencias es una propuesta teórico-metodológico que dota a la práctica de Gestión de Recursos Humanos (GRH) de una noción holística e integradora sobre el desempeño de las personas en el trabajo, en la cual se debe superar necesariamente la concepción taylorista; y a nivel metodológico demanda la creación de técnicas que reconstruyan la interacción compleja que existe entre las características del individuo y las características de la situación de trabajo en la organización.

Los perfiles de competencias de puestos superan a los tradicionales perfiles de cargo o profesiogramas que comprenden funciones descritas en un plano puramente cognitivo. Aquí, el clásico contenido de trabajo del puesto expresado en funciones o tareas, es superado por las competencias. Según la NC 3000:2007 define el perfil de competencias como:

Documento que describe las competencias requeridas para un cargo y expresa la relación de los objetivos estratégicos y metas con las capacidades que debe desarrollar el personal de la organización. Dicho perfil se expresa en un lenguaje de resultados claves y no comprende ni describe necesariamente todas las funciones y tareas del cargo, solo se centra en aquellos elementos fundamentales.

La gestión del desempeño por competencias se enfoca esencialmente en el desarrollo, en lo que las personas "serán capaces de hacer" en el futuro, por lo que el pensamiento estratégico y la proactividad le son inmanentes. La gestión de competencias es hoy concepción relevante a comprender en la GRH, implicando mayor integración entre estrategia, sistema de trabajo y cultura organizacional, junto a un conocimiento mayor de las potencialidades de las personas y su desarrollo (Cuesta, 2000).

La selección de personal es un proceso que es realizado mediante concepciones y técnicas efectivas, consecuente con la dirección estratégica de la organización y las políticas de GRH derivadas, con el objetivo de encontrar al candidato que mejor se adecue a las competencias presentes y futuras previsibles de un puesto y de una empresa concreta o específica.

Los perfiles de competencias definidos por las organizaciones para sus puestos o cargos, son esencialmente conjuntos de competencias secundarias (holísticas a plenitud), y van con descripciones más o menos detalladas de pautas de conductas (dimensiones) que ejemplifican el desarrollo de una competencia. Es importante la consideración actual de las competencias "futuras previsibles", pues las demandas de la altísima dinámica empresarial de hoy hacen superar el concepto de "seleccionar para un puesto determinado", y más bien se aprecia 
al puesto en su evolución en relación con otros puestos significándose así la flexibilidad o polivalencia necesaria, y no sólo respondiendo las competencias del contenido del puesto sino también a las comprendidas por las relaciones interpersonales eficaces (priorizándose las habilidades de laborar en equipos) y la cultura organizacional.

La existencia y actualidad del perfil de competencias es determinante para el proceso de selección. En esta fase se deciden los indicadores o parámetros (dimensiones) que servirán de referencia o patrón para comparar las características (competencias) medidas o valoradas en el aspirante al puesto, precisamente obtenidos del perfil de competencias. Es imprescindible atender a la existencia y actualidad de tal perfil. Si no existe o no se encuentra actualizado, habrá que recurrir al análisis y diseño de puesto o cargo de trabajo (Cuesta, 2000).

La investigación que se presentase centra por tanto, en el estudio de los elementos necesarios (tareas, condiciones, exigencias, competencias laborales, calidad de vida laboral) para analizar el cargo de Técnico A en Gestión de Recursos Humanos en la Dirección General de Recursos Humanos (DGRH) de la Oficina del Historiador de la Ciudad de La Habana $(\mathrm{OHCH})$, con el objetivo de crear el perfil de competencias y una propuesta de selección de personal para este cargo. Esto contribuye a elevar la calidad del proceso de reclutamiento, selección y desarrollo de este puesto de trabajo, así como a aumentar la eficacia y eficiencia de dicha organización laboral.

La $\mathrm{OHCH}$ es un organismo adjunto al Consejo de Estado, que tiene la responsabilidad de fomentar la cultura habanera, nacional y sus vínculos internacionales, invirtiendo en la conservación de los monumentos históricos y bienes de La Habana que corresponden al patrimonio nacional. Dentro de esta organización, la DGRH tiene como misión garantizar el reclutamiento, la selección, capacitación y desarrollo de los recursos humanos en un ambiente seguro y con un sistema de compensación adecuado. Es en esta Dirección donde labora el Técnico A en Gestión de Recursos Humanos, quien se encarga particularmente de los procesos de reclutamiento y selección de personal para todas las entidades pertenecientes a la $\mathrm{OHCH}$. Este cargo resulta fundamental, pues provee la fuerza de trabajo de toda la organización, y es el primer trabajador con quien se comunica la persona que solicita ingresar a la bolsa de empleo.

\section{Metodología}

\section{Muestra}

En la investigación se contó con la participación de 8 sujetos, de los cuales 3 fueron informantes clave y 5, expertos en el cargo objeto de estudio. 


\section{Informantes clave}

Como criterios de selección se tuvo en cuenta que: i.) Ocuparan un cargo de Directivo de o Especialista de Recursos Humanos en la DGRH; ii.)Poseer como mínimo 1 año de experiencia en este cargo; iii.) No formaran parte de la muestra de expertos.

Partiendo de estos requisitos, participaron como informantes clave: el Director de Empleo y Salario (25 años de experiencia), el Especialista Principal de Inspectores-investigadores (5 años de experiencia), y un Técnico A en Gestión de Recursos Humanos (2 años de experiencia).

\section{Expertos en el puesto de trabajo}

Como criterios de selección se tuvo en cuenta que: i.)Ocuparan el cargo objeto de estudio o fueran su jefe inmediato superior; ii.)Poseer como mínimo 1 año de experiencia en el cargo Técnico A en Gestión de Recursos Humanos; iii.) Poseer buenos resultados de trabajo (a partir de evaluaciones de desempeño anteriores y criterio del jefe inmediato superior).

Partiendo de estos requisitos, participaron como expertos: cuatro Técnicos A en Gestión de Recursos Humanos (entre 3 y 8 años de experiencia) y el Subdirector de Reclutamiento y Selección (1 año de experiencia).

\section{Procedimiento de la investigación}

Para la realización del presente estudio se empleó una metodología de corte mixto, integrando la información obtenida mediante varias técnicas y métodos tanto cualitativos como cuantitativos. La aplicación de técnicas y análisis de la información se dividió en tres etapas fundamentales:

\section{Etapa 1. Caracterización de la organización}

En un primer momento se aplicaron entrevistas semi-estructuradas a la muestra de informantes claves, con el objetivo de elaborar una Guía de Información General de la Organización (Localización, Misión, Visión, Funciones, Historia, Personal, etc.) y la Caracterización de la Función de Recursos Humanos en la misma (Planeamiento, Reclutamiento, Selección, Inducción).

Se realizó también un análisis de documentos existentes en la organización que ofrecían información relevante para el estudio que se realiza. Los documentos consultados fueron: Planeación estratégica, Organigrama, Plantilla de personal, Profesiogramas o calificadores de cargos, Manuales de normas y procedimientos del cargo Técnico A en Gestión de Recursos Humanos, Guía de control interno y prevención de riesgos y Manual de Seguridad y Salud en el Trabajo. 


\section{Etapa 2. Caracterización del puesto de trabajo}

Como segundo momento fueron aplicadas varias técnicas cualitativas a la muestra de expertos en el puesto de trabajo, con el fin de caracterizar las condiciones, exigencias y competencias necesarias para el mismo. Las técnicas aplicadas fueron:

2.1. Entrevista de incidentes críticos. Entrevista estructurada que permite identificar situaciones laborales críticas y las competencias del sujeto evidenciadas en el repertorio de comportamientos que éste ha desplegado en su actuación como titular del cargo; fundamentalmente a través del relato de experiencias vividas (positivas y negativas) durante su trabajo en dicho cargo. Estos criterios permiten diferenciar cualitativamente un desempeño promedio de un desempeño superior o competente.

2.2. Diario de actividades. Enumeración de las operaciones que se desarrollan en el cargo estudiado y el período de tiempo en que se realizan.

2.3. Modelo de condiciones laborales. Cuestionario que permite describir las condiciones internas y externas del cargo, así como el carácter de su influencia en los resultados del trabajo, utilizando como referente la clasificación de Straub y Hacker (Schaarschmidt y Prado, 2002). Este fue aplicado a manera de entrevista.

A partir de la información recogida en estas técnicas, se elaboró un Diagrama de exigencias de la actividad laboral. Esta técnica permitió identificar las exigencias laborales de tipo 1, 2 y 3 del cargo a partir de la clasificación propuesta por Quas y Raum (Schaarschmidt y Prado, 2002), así como su interinfluencia.

\section{Etapa 3. Determinación de las competencias clave para el cargo}

Como tercer momento de la aplicación, se confeccionó un listado de competencias claves y dimensiones para el desempeño superior (exitoso) en el cargo a partir de la información recogida en la etapa anterior. Este listado se elaboró a partir de la clasificación propuesta por Mertens (2000), y fue tomado para la aplicación de la siguiente técnica:

3.1. Método MiniDelphi. Es una adaptación del conocido Método Delphi elaborada por Cuesta (2000) que permite validar las competencias y dimensiones propuestas, a través del consenso de la muestra de expertos. La aplicación de este método requirió una previa y breve capacitación al grupo de expertos en los términos conceptuales-teóricos utilizados en las técnicas.

\section{Resultados}

Partiendo del análisis del Método MiniDelphi, fueron identificadas cinco competencias clave para el desempeño exitoso en el cargo de Técnico A en Gestión 
de Recursos Humanos, las cuales coincidieron con las propuestas inicialmente por el equipo de investigadores y fueron reconocidas por la muestra de expertos con un elevado por ciento de consenso (80\%-100\%) (Ver Anexo 1).

Estas competencias fueron ponderadas en una segunda fase de análisis según su nivel de importancia en el desempeño exitoso del cargo, obteniéndose un ordenamiento jerárquico de las mismas con un por ciento de consenso siempre mayor al 60\% (Ver Anexo 2).

Finalmente en la tercera y cuarta fase de análisis, fueron validadas las dimensiones competenciales y los indicadores comportamentales respectivamente. En ambas fases se obtuvo siempre un por ciento de consenso mayor al $60 \%$, excepto en un indicador comportamental, el cual fue excluido del perfil por competencias (Ver Anexo 3).

A partir de los resultados fue confeccionado el Modelo de Perfil de Competencias para el cargo de Técnico A en Gestión de Recursos Humanos, en cual se presentan las competencias clave según su nivel de importancia en el desempeño exitoso del cargo (ponderación obtenida en la fase 2 del análisis).

TABLA 1.

Perfil de competencias del cargo Técnico A en Gestión de Recursos Humanos

\section{Perfil del puesto de trabajo}

Descripción del puesto de trabajo.

Denominación del Cargo: Técnico A en Gestión de Recursos Humanos

Unidad Organizativa a la cual pertenece: Dirección General de Recursos Humanos de la Oficina del Historiador de la Ciudad de La Habana.

Dependencia Jerárquica Superior: $\mathrm{OHC}$

Categoría Ocupacional: Técnico

Proceso fundamental en el que se desempeña:

Subprocesos fundamentales en los cuales se desempeña:
Grupo Escala: VIII

Reclutamiento y Selección de personal.

Acometer acciones a partir de los lineamientos de organización del trabajo, empleo y capacitación; Tramitar solicitudes de sus entidades con los organismos y niveles correspondientes; Ejecutar los programas de selección de la fuerza de trabajo;

Estudiar los aspectos socio-psicológicos de la fluctuación de la fuerza de trabajo;

Planificar, organizar y ejecutar las comprobaciones y demás tareas sobre los candidatos a ingresar a la entidad;

Realizar otras funciones de similar naturaleza según se requiera. 
Misión del Puesto: Reclutamiento y selección de personal de las entidades pertenecientes a la Oficina del Historiador de la Ciudad de La Habana.

Principales Funciones y Tareas:

Entrega y recogida de planillas de solicitudes de ingreso.

Chequeo de los datos de la planilla.

Organización de la información en los archivos y procesamiento estadístico.

Preparar el modelo de investigación para el investigador con los datos de la persona.

Clasificar los expedientes.

Realizar el proceso de conciliación.

Ingreso a los candidatos: Entrevistas, chequeo médico, seguimiento a las entrevistas de los candidatos con las entidades, ver que la plaza que piden realmente existe y está vacante.

Confeccionar las cartas de presentación.

Archivar las bajas y aquellas que llevan más de 5 años eliminarlas.

Principales Recursos Materiales asignados para desempeñar sus Funciones:

Medios de Transporte: Transporte colectivo.

Medios de Comunicación: Teléfono, correo electrónico.

Medios Ofimáticos: PC de escritorio, impresora, red, escáner.

Muebles de Oficina: Buró, mesa de computadora, silla rotatoria, archivos.

Medios de Climatización: Aire acondicionado.

\section{Responsabilidades:}

Materiales.

Imagen Organizacional.

Calidad Final del Producto.

Condiciones de Trabajo óptimas para el desempeño de la actividad:

Horario de Trabajo: lunes a jueves de 8:00 am - 5:20 pm, viernes de 8:00 am - 5:00 pm con

registro de hora de entrada y salida, sin remuneración por las horas extras.

Ambiente de Trabajo:

Condiciones estándares de oficina.

Esfuerzo físico y mental necesario: Actividad intelectual media. Esfuerzo físico bajo.

Riesgos y Enfermedades Profesionales a las que está expuesto:

Caídas desde un mismo nivel o desde escaleras,

Golpes con objetos por hacinamiento,

Enfermedades relacionadas con el uso de medios de cómputo (Síndrome del túnel carpiano),

Stress.

Medios de Protección Necesarios:

Mantener nivel de iluminación de 300 lux en el archivo y 500 lux para la escritura, mecanografía, lectura y procesamiento de datos.

La exposición a niveles de presión sonora debe ser menor de $65 \mathrm{~dB}(\mathrm{~N})$.

El mobiliario de oficina debe ser ergonómico, con la altura y el confort adecuado.

\section{Requisitos mínimos personales}

Dimensiones de los requisitos personales.

\begin{tabular}{ll}
\hline Cargo nivel académico & Graduado de Nivel Medio Superior. \\
\hline Especialidades preferentes & $\begin{array}{l}\text { Preferiblemente en una especialidad afín a } \\
\text { los Recursos Humanos. }\end{array}$ \\
\hline
\end{tabular}


Experiencia en el tema

Formación
Nociones elementales sobre Recursos Humanos (cómo llenar las planillas, trabajar con los archivos, realización de entrevistas, etc.)

Tener entrenamiento en el puesto de trabajo y dominio de la legislación laboral vigente. Poseer conocimientos en la informática y de gestión de bases de datos.

\section{Competencias para el puesto}

Nombre de la competencia: Capacidad de organización.

Nivel de desarrollo óptimo de la competencia para el cargo: Experto.

\section{Definición de la competencia}

Capacidad para determinar de forma eficaz, fases, etapas, metas y prioridades para la consecución de objetivos, a través del desarrollo de planes de acción, incluyendo los recursos necesarios y los sistemas de control.

\begin{tabular}{ll}
\hline Dimensiones & Indicadores comportamentales \\
\hline Administración del tiempo & Establece prioridades en las actividades laborales. \\
& Establece plazos y fijar responsabilidades. \\
& Planifica estrategias que minimizan el tiempo de la \\
actividad y optimizan el desarrollo de las tareas. & Cumple los plazos establecidos, ajustándose a los \\
sistemas y esquemas de trabajo indicados.
\end{tabular}

Control del trabajo realizado

Establece mecanismos de control y medición de los resultados.

Evalúa los resultados y establece acciones concretas a seguir.

Realiza el trabajo en etapas, acciones y tareas, controlando la asignación de los recursos necesarios y aplicando acciones correctivas cuando se requiere. Participa en los espacios y ambientes creados para la retroalimentación sobre las tareas en marcha. Da respuestas rápidas y efectivas ante las diferentes situaciones que se le presenten.

Multifuncionalidad

Es capaz de dirigir varios proyectos

simultáneamente, sin perder el control de ninguno de ellos.

Conoce de manera global la gestión de la entidad. Se responsabiliza con varios de los procesos claves de la entidad.

Ejecuta los procesos y tareas de acuerdo a la planificación.

Diseñar e implementar estrategias

Aporta en el proceso de diseño de las estrategias y objetivos de la entidad

Participa en el diseño y ejecución de acciones de control y seguimiento a la implantación de la estrategia diseñada Ejecuta el plan de acciones que permite evaluar el cumplimiento a la estrategia. 
Nombre de la competencia Comunicación eficiente y persuasiva.

Nivel de desarrollo óptimo de la competencia para el cargo: Experto.

\section{Definición de la competencia}

Capacidad para generar y mantener un flujo de comunicación adecuado entre los miembros de la organización y los candidatos que allí asisten, utilizando los medios de comunicación establecidos para cada caso, favoreciendo así el establecimiento de relaciones laborales sanas y beneficiosas.

\section{Dimensiones competenciales}

Dimensiones competenciales

Aptitud verbal

Aptitud verbal

Escucha

Ofrecer respuestas

\section{Descriptivos comportamentales}

Posee una dicción apropiada para la comunicación. Se expresa eficazmente dando muestras de manejar un lenguaje técnico y profesional.

Emplea un tono natural y espontáneo.

Formula argumentos acorde a su nivel de formación y experiencia, así como del nivel del cargo que ocupe. Se expresa de manera que incida e impacte directamente en el interlocutor o candidato de la organización.

Posee una disposición para recibir cualquier tipo de información, queja o sugerencia que provenga del candidato.

Se preocupa por escuchar y satisfacer las necesidades de sus candidatos.

Tiene la capacidad para comprender la información recibida y ofrecer en correspondencia una respuesta.

Presta atención a señales verbales y no verbales del emisor para reaccionar de la forma más adecuada.

Se expresa con claridad, utilizando un vocabulario adecuado.

Comparte información, expresándose con fluidez y realizando explicaciones claras y precisas.

Adapta sus respuestas a las características del interlocutor al que se destinan.

Se pone en el lugar del otro, logrando entender empáticamente el estado del candidato y aprovechar la facilidad de sintonía para hacerse entender en sus comunicaciones y lograr actitudes de cambio en estos. Muestra una actitud y predisposición para captar las necesidades de los candidatos.

Negocia bajo una política de gana-gana, forjando relaciones de entendimiento mutuo que supongan ventajas para ambas partes, creando sinergia enfocada al logro de objetivos mutuos y ventajosos. 
Persuasión

Expresa ideas o hechos claramente y de una manera persuasiva.

Convence a los otros del punto de vista propio.

Influye o impresiona a los candidatos para que contribuyan

a alcanzar los objetivos propios y de la organización.

Logra causar un efecto específico en el candidato, una impresión determinada o una actuación concreta según el objetivo que se persigue.

Utiliza estrategias que den respuesta a cada situación

o factor en concreto, basándose en los argumentos

estratégicos de la empresa como estrategia de persuasión.

Nombre de la competencia

Comportamiento Ético.

Nivel de desarrollo óptimo de la competencia para el cargo: Experto.

\section{Definición de la competencia}

Capacidad para comportarse acorde a las directrices, normas y valores, organizacionales y del sector de selección del personal de la Oficina del Historiador de la Ciudad de La Habana, dando muestras de un elevado nivel de compromiso, así como de profesionalidad y confiabilidad en el actuar.

\section{Dimensiones competenciales Descriptivos comportamentales}

Integridad

Interioriza y actúa acorde a directrices, normas y valores organizacionales y del sector de selección del personal de la oficina del Historiador de la Ciudad de La Habana.

Se comporta de acuerdo a las normas éticas y morales socialmente aceptadas.

Comunica las intenciones, ideas y sentimientos abierta y directamente tanto a sus superiores como a sus compañeros de trabajo.

Asume correctamente el rol del profesional en el trato con el público, incluso ante cualquier dificultad.

Confiabilidad

Vela por los intereses y recursos que están bajo su custodia. Dar cuentas sobre los mismos.

Realiza el mejor uso de los recursos económicos, financieros, técnicos, humanos.

Muestra total discreción en el manejo la información sensible y confidencial. 
Profesionalismo

Compromiso organizacional
Actúa conforme a las normas de trabajo y a los estándares técnicos establecidos.

Se responsabiliza de las consecuencias negativas de la propia actuación.

Orienta los intereses personales hacia las necesidades, prioridades y objetivos de la organización, al tener claramente definida la primacía del bien colectivo sobre los intereses particulares.

Sacrifica o subordina intereses personales que perjudiquen, de manera significativa, los objetivos organizacionales.

Se esfuerza permanentemente hacia la consecución de los objetivos planteados por la oficina.

Da muestras de lealtad y sentido de pertenencia hacia la organización, en tanto la defiende y difunde sus intereses.

\section{Nombre de la competencia: Autocontrol emocional.}

Nivel de desarrollo óptimo de la competencia para el cargo: Avanzado.

\section{Definición de la competencia}

Capacidad de mantener las propias emociones bajo control y evitar reacciones negativas ante provocaciones, oposición u hostilidad por parte de otros o bajo condiciones de estrés.

\section{Dimensiones competenciales Descriptivos comportamentales}

Tolerancia a las opiniones de los entrevistados
Mantiene relaciones cordiales en el trato con los candidatos internos y externos de la institución. Se establece una comunicación empática y asertiva que conlleva a la prestación de servicios serios, precisos y efectivos. Controla sus impulsos y emociones, propone acciones para el manejo efectivo de las situaciones que no poseen una buena estructuración.

Promueve el manejo de la tolerancia y efectividad entre sus pares.

Mantiene una relación profesional con sujetos que no se expresan de manera correcta.

Reitera la información, las veces que sean necesarias a los entrevistados cuando estos no han comprendido, siendo capaz de mantener una comunicación cordial. 
Manejo de conflictos
Argumenta su posición, dando elemento que le permitan hacer valer su criterio ante criterios divergentes. Maneja con profesionalismo las situaciones conflictivas tanto con los candidatos externos como con los propios compañeros de trabajo.

Es asertivo cuando la posición contraria posee los argumentos más contundentes, en situaciones conflictivas. Mantiene sereno y firme en situaciones de tensión. Puede discernir, evaluar con objetividad y tomar decisiones efectivas en escenarios caracterizados por el riesgo, la incertidumbre y complejidad.

Distanciamiento emocional

Mantiene una distancia objetiva ante situaciones emotivas negativas de los entrevistados.

Mantiene al margen los problemas personales en la realización del trabajo.

\section{Nombre de la competencia Gestión de información}

Nivel de desarrollo óptimo de la competencia para el cargo: Avanzado.

\section{Definición de la competencia}

Capacidad para saber cuándo y por qué se necesita información, qué alcance y nivel de complejidad requiere, dónde encontrarla, cómo evaluarla, utilizarla y comunicarla de manera tal que impacte positivamente en la toma de decisión de usuarios durante procesos de selección de personal, en el funcionamiento de la organización y en la optimización de los servicios a terceros.

\section{Dimensiones competenciales Descriptivos comportamentales}

Búsqueda de la Información Aplica técnicas de lectura en función de las circunstancias y los requerimientos.

Emplea las tecnologías informáticas con eficiencia.

Actualiza la información que se maneja.

Revisa la información que entregan los candidatos a la bolsa.

Análisis de la Información

Procesa la información recogida.

Ordena dicha información.

Propone variantes para la toma de decisiones en función de los resultados de sus análisis.

Comparte y socializa la información y los resultados de los análisis presentándolos adecuadamente a sus superiores y otras entidades.

Redacta adecuadamente, sin deficiencias ortográficas, emplea citas y referencias y presta atención al detalle. 
Conoce y aplica las políticas y medidas de seguridad de la información.

Mantiene actualizados los archivos con las informaciones más recientes.

Registra y recupera adecuadamente la información.

\section{Modelo para Programa de Selección de Personal}

Partiendo del perfil de competencias expuesto anteriormente, se elaboró una propuesta de diseño del Modelo de Selección del Personal para el cargo Técnico A en Gestión de Recursos Humanos, el cual se expone a continuación.

Test de estilo de comunicación.

Esta prueba tiene como objetivo determinar el estilo de comunicación predominante en el individuo, clasificándolo básicamente en cuatro estilos o rasgos: comunicación orientada hacia la acción, hacia los procesos, hacia las personas o hacia las ideas. La calificación del test se realiza agrupando los ítems con el estilo de comunicación que le corresponde según lo predeterminado por el test, y determinando cuál es el estilo predominante según el que mayor cantidad de ítems posea. El estilo comunicativo necesario para desempeñarse exitosamente y ser eficiente en el cargo de Técnico A en Gestión de Recursos Humanos, es el orientado hacia las personas, que incluye rasgos como: espontaneidad, empatía y receptividad a las solicitudes del candidato.

\section{Test de Empleo del tiempo}

Este test tiene como objetivo el valorar las habilidades del sujeto en el empleo del tiempo para la realización de disímiles actividades. La prueba posee 25 ítems y se responde mediante la elección de las opciones que más se adecúan a las características del candidato (por lo general, a veces o nunca). La calificación del test se hace asignando puntuaciones a las diferentes respuestas, y ubicando la puntuación final en una escala. Para un desempeño superior en el cargo de Técnico A en Gestión de Recursos Humanos, es necesario que el candidato quede ubicado en el último intervalo (80-100 puntos), el cual describe una adecuada administración del tiempo y control de situaciones.

\section{Test de comportamiento ético}

Constituye una técnica proyectiva de completamiento que combina material pictórico y verbal, y que está inspirada en el Test de frustración de Rosenzweig. A partir de esto, se le presentan al sujeto una serie de dibujos previamente diseñados 
en los que se describe a un personaje principal que se encuentra envuelto en una situación ética, es decir, una situación donde debe tomar una decisión con implicaciones morales. Se dan instrucciones al sujeto para que escriba en el espacio que se deja en blanco lo que el personaje contestaría en esa situación, instándosele para que de la primera respuesta que le acuda a la imaginación. La evaluación de este test se basa en el presupuesto de que el sujeto evaluado se identifica con el personaje de cada lámina, y proyecta sus propios valores éticos y morales en la respuesta que da.

\section{El juego de roles (role-playing)}

La técnica del juego de roles consiste en preparar una situación, en la que uno o más participantes representan personajes y aspectos de una actividad laboral, actuando según el papel que se les ha asignado previamente. El objetivo de la técnica estaría orientado a evaluar las competencias: gestión de información, autocontrol emocional y comunicación eficiente y persuasiva. La situación concreta propuesta para la evaluación del cargo consistiría en: Se presenta un candidato ya perteneciente a la bolsa de la Entidad Empleadora, que desea actualizar su expediente con el ingreso de nuevos documentos al mismo (gestión de información). En los documentos presentados por el candidato existe una irregularidad, por la cual este debe intentar colocarla en una situación emocional comprometedora (autocontrol emocional) con el objetivo de que esta acepte el documento, o incluso que le otorgue una plaza de trabajo. Finalmente debe ser evaluada la capacidad de la técnico para persuadir al candidato (comunicación eficiente y persuasiva).

\section{Entrevista}

Se puede definir como una entrevista semi-estructurada, focalizada en la obtención de ejemplos conductuales de la vida laboral, académica y/o personal del entrevistado, susceptibles de ser utilizados como predictores de sus comportamientos futuros en el trabajo. Este tipo de entrevista parte del principio de que el mejor predictor de la conducta futura, es la conducta pasada; es decir, si una persona ha sido capaz de desarrollar un comportamiento en el pasado, en similares circunstancias, podrá volver a repetirlo.

Requiere que el entrevistador formule preguntas sobre hechos específicos del pasado del sujeto y que pongan de manifiesto que el participante ha puesto en práctica los comportamientos incluidos en cada competencia que se desea evaluar, en este caso, las competencias de gestión de información, autocontrol emocional, comportamiento ético, capacidad de organización y comunicación eficiente y persuasiva (con sus respectivos indicadores comportamentales). 


\section{Conclusiones}

Las principales funciones de la Dirección General de Recursos Humanos de la Oficina del Historiador de la Ciudad de La Habana, son: verificar a las personas que soliciten ingresar a la Bolsa de Empleo; elaborar Expediente de Candidatos; ofertar la Fuerza de Trabajo solicitada; aprobar aquellos Candidatos que reúnan los requisitos de idoneidad y velar porque la Fuerza de Trabajo esté calificada.

Las condiciones laborales para el cargo estudiado son adecuadas; sin embargo, existe algunas deficiencias en las mismas, encontrándose que en las condiciones exteriores se presentan insuficiencias en las condiciones ambientales (hacinamiento, edificación en mal estado, iluminación artificial y mal distribuida en la habitación, y déficit de ciertos materiales de oficina). Mientras que en las condiciones interiores existen algunas disfunciones en las premisas de rendimiento psicofísicas actuales, evidenciándose en los trabajadores: desmotivación por sobrecarga de trabajo, insatisfacción con las condiciones laborales exteriores, estado emocional ambivalente debido a las deficiencias que se describen, y fatiga como un estado disfuncional provocado por la actividad laboral.

Las exigencias laborales para el cargo estudiado, son: Exigencias tipo I: Reclutamiento y selección del personal de la Oficina del Historiador de la Cuidad de La Habana. Exigencias tipo II: recogida de planillas de solicitudes de ingreso, chequeo de los datos de la planilla, organización de la información en los archivos y procesamiento estadístico, preparar el modelo de investigación para el investigador con los datos de la persona, y aclararlos bien al investigador, clasificar los expedientes, proceso de conciliación, ingreso a los candidatos: entrevistas, chequeo médico, seguimiento a las entrevistas de los candidatos con las entidades, ver que la plaza que piden realmente existe y está vacante, hacer cartas de presentación, archivar las bajas y las que llevan más de 5 años eliminarlas. Como Exigencias tipo III: habilidad en el procesamiento y registro de información, actitud favorable hacia el trabajo con el público, capacidad de autocontrol emocional, capacidad de comportarse de forma ética, habilidad para gestionar información, habilidad de comunicarse de modo eficiente y persuasivo, capacidad de organización y planificación.

\section{Nota}




\section{Referencias bibliográficas}

Chiavenato, I. (2001). Gestión del talento humano. El papel de los recursos humanos en la organización. Editorial Mc Graw Hill. Madrid, España.

Cuesta, A. (2000). Gestión de competencias: Monografía. Versión digital.

McClelland, D. C. y Boyatzis, R.E. (1982) Leadership motive pattern and long-term success in management. Journal of Applied Psychology, 67, pp. 737 - 43.

Mertens, L. (2000) La gestión por competencias en la empresa y formación profesional. Editorial OEI, España.

Schaarschmidt, U. y de Prado, R. (2002). Introducción a la Psicología de Trabajo. Tomo I. Editorial Félix Varela. La Habana, Cuba.

\section{Anexos}

\section{ANEXO 1.}

Resultados de la Primera Fase de Análisis del Método MiniDelphi

(Determinación de las competencias clave para el cargo)

\begin{tabular}{ccccccc}
\hline Competencias & Experto 1 & Experto 2 & Experto 3 & Experto 4 & Experto 5 & Cc (\%) \\
\hline $\begin{array}{c}\text { Gestión de } \\
\text { información }\end{array}$ & si & si & si & si & si & 100 \\
\hline $\begin{array}{c}\text { Autocontrol } \\
\text { emocional }\end{array}$ & si & si & si & si & si & 100 \\
\hline $\begin{array}{c}\text { Comportamiento } \\
\text { Ético }\end{array}$ & si & si & si & si & si & 100 \\
\hline $\begin{array}{c}\text { Comunicación } \\
\text { eficiente y } \\
\text { persuasiva }\end{array}$ & no & si & si & si & si & 80 \\
\hline $\begin{array}{c}\text { Capacidad de } \\
\text { organización }\end{array}$ & si & si & si & si & no & 80 \\
\hline
\end{tabular}




\section{ANEXO 2}

Resultados de la Segunda Fase de Análisis del Método MiniDelphi (Ponderación de las competencias clave para el cargo)

\begin{tabular}{lcccccccc}
\hline Competencias & E1 & E2 & E3 & E4 & E5 & Rj & Rjm & $\begin{array}{l}\text { Cc } \\
(\%)\end{array}$ \\
\hline Gestión de información & 5 & 4 & 5 & 3 & 5 & 22 & 4,4 & 60 \\
\hline Autocontrol emocional & 3 & 5 & 3 & 5 & 3 & 19 & 3,8 & 60 \\
\hline Comportamiento ético & 4 & 3 & 4 & 4 & 1 & 16 & 3,2 & 60 \\
\hline $\begin{array}{l}\text { Comunicación eficiente y } \\
\text { persuasiva }\end{array}$ & 2 & 2 & 2 & 2 & 2 & 10 & 2 & 100 \\
\hline Capacidad de organización & 1 & 1 & 1 & 1 & 4 & 8 & 1,6 & 80 \\
\hline
\end{tabular}

\section{ANEXO 3}

Resultados de la Tercera Fase de Análisis del Método MiniDlephi (Determinación de las Dimensiones Competenciales)

\begin{tabular}{|c|c|c|c|c|c|c|c|}
\hline Competencias & Dimensiones Competenciales & $\mathrm{E} 1$ & E2 & E3 & E4 & E5 & $\begin{array}{l}\mathrm{Cc} \\
(\%)\end{array}$ \\
\hline \multirow{3}{*}{$\begin{array}{l}\text { Gestión de } \\
\text { información }\end{array}$} & Búsqueda de la Información & si & si & si & si & si & 100 \\
\hline & Análisis de la Información & si & si & si & si & si & 100 \\
\hline & Control de la Información & si & si & no & si & si & 80 \\
\hline \multirow{3}{*}{$\begin{array}{l}\text { Autocontrol } \\
\text { emocional }\end{array}$} & $\begin{array}{l}\text { Tolerancia a las opiniones de los } \\
\text { entrevistados. }\end{array}$ & si & si & no & si & si & 80 \\
\hline & Manejo de conflictos. & si & si & si & si & no & 80 \\
\hline & Distanciamiento emocional & si & si & si & si & si & 100 \\
\hline \multirow{4}{*}{$\begin{array}{l}\text { Comportamiento } \\
\text { Ético }\end{array}$} & Integridad. & si & $\mathrm{si}$ & no & $\mathrm{si}$ & si & 80 \\
\hline & Confiabilidad & si & si & si & si & si & 100 \\
\hline & Profesionalismo. & si & $\mathrm{si}$ & no & si & $\mathrm{si}$ & 80 \\
\hline & Compromiso organizacional. & si & si & si & $\mathrm{si}$ & si & 100 \\
\hline \multirow{4}{*}{$\begin{array}{l}\text { Comunicación } \\
\text { eficiente y } \\
\text { persuasiva }\end{array}$} & Aptitud verbal & si & si & si & si & si & 100 \\
\hline & Escucha & si & si & si & si & si & 100 \\
\hline & Ofrecer respuestas & si & $\mathrm{si}$ & $\mathrm{si}$ & $\mathrm{si}$ & si & 100 \\
\hline & Persuasión. & si & si & si & si & si & 100 \\
\hline \multirow{4}{*}{$\begin{array}{l}\text { Capacidad de } \\
\text { organización }\end{array}$} & Administración del tiempo & si & $\mathrm{si}$ & $\mathrm{si}$ & $\mathrm{si}$ & $\mathrm{si}$ & 100 \\
\hline & Control del trabajo realizado & si & si & si & si & si & 100 \\
\hline & Multifuncionalidad & si & si & si & si & si & 100 \\
\hline & Diseñar e implementar estrategias & no & si & no & si & si & 60 \\
\hline
\end{tabular}




\section{(c) (i) (2)(2)}

Esta obra está bajo una licencia de Creative Commons Reconocimiento - NoComercial - CompartirIgual (by-nc-sa) 